\title{
A retrospective study of hyponatremia in tetraplegic/paraplegic patients with a review of the literature
}

\author{
B M Soni MBBS MS, ${ }^{1}$ S Vaidyanthan MS MCh PhD,${ }^{2} \mathrm{~J}$ W H Watt FFARCS, ${ }^{3}$ \\ K R Krishnan MB FRCS ${ }^{4}$
}

\begin{abstract}
${ }^{1}$ Associate Specialist, ${ }^{2}$ Registrar, ${ }^{3}$ Consultant Anaesthetist, ${ }^{4}$ Director, Mersey Regional Spinal Injuries Centre, District General Hospital, Town Lane, Southport, Merseyside, PR8 6PN, England.
\end{abstract}

The aetiology of hyponatremia in tetraplegic patients is multifactorial and includes not only general factors such as the use of diuretics and the intravenous infusion of hypotonic fluids, but also certain mechanisms which operate in the spinal cord injured: decreased renal water excretion due to both intrarenal and arginine vasopressin dependent mechanisms (resetting of the osmostat), coupled with habitually increased fluid intake, and the ingestion of a low salt diet. Between 1984 and 1993 we treated 28 episodes of hyponatremia in 19 patients (males: 10; females: 9). Fourteen were tetraplegic and five paraplegic (thoracic lesion in four and lumbar lesion in one). Six patients were asymptomatic during seven episodes of hyponatremia which were detected during routine blood tests. Seven patients were suffering from an acute chest infection, three had an acute urinary tract infection, one had an infected ischial pressure sore and a 69 year old paraplegic patient had bronchopneumonia as well as sepsis from a gangrenous pressure sore in the supraanal region. The time interval between the onset of paralysis and occurrence of the first episode of hypnoatremia was less than a month in only four of the patients. The lowest plasma sodium level observed was less than $100 \mathrm{mmol} / \mathrm{l}$ in two, between 100 and $110 \mathrm{mmol} / \mathrm{l}$ in four, between 111 and $120 \mathrm{mmol} / \mathrm{l}$ in eight patients, and between 121 and $128 \mathrm{mmol} / \mathrm{l}$ in 14 cases. Six patients also had hypokalemia $\left(\mathrm{K}^{+}<3 \mathrm{mmol} / \mathrm{l}\right)$. Only one patient had an elevated plasma creatinine $(201 \mathrm{umol} / \mathrm{l})$. Treatment of sepsis and fluid restriction were the mainstay of treatment with only two patients receiving hypertonic saline. All patients with underlying sepsis were treated with antibiotics, usually administered intravenously. The outcome was good in 26 of the 28 episodes.

Two patients died: a 68 year old tetraplegic patient with consolidation of the left lung, cystadenocarcinoma of both ovaries and squamous cell carcinoma of the forehead who presented with generalised oedema, with a plasma sodium level of $118 \mathrm{mmol} / \mathrm{l}$, and potassium of $2.4 \mathrm{mmol} / \mathrm{l}$ and who was treated with $2 \mathrm{~N}$ saline + postassium + frusemide; she died 1 day later. The only other death was that of a 78 year old female tetraplegic patient who 2 days after sustaining cervical trauma developed hyponatremia because of intravenous infusion of hypotonic fluids given at another hospital, presumably to correct hypotension. She recovered from hyponatremia with fluid restriction, but 3 days later she succumbed to bronchopneumonia and respiratory insufficiency. No patient developed central pontine myelinolysis. No patient with a severe degree of hyponatremia (sodium $<100 \mathrm{mmol} / \mathrm{l}$ ) had respiratory involvement requiring ventilatory assistance. In conclusion, hyponatremia is seen in tetraplegic patients often in association with sepsis either in the lungs or in the urinary tract, and is best managed by treament of the predisposing factor(s) along with fluid restriction. 


\section{Introduction}

Hypoosmolar hyponatremia has been observed in spinal cord injury patients with a prevalence rate of approximately $5-10 \%$ (serum or plasma sodium of less than $130 \mathrm{mmol} / \mathrm{l}) .{ }^{1}$ Apart from the aetiological factors which cause hyponatremia in the general population, such as diuretics and intravenous infusion of hypotonic fluids, ${ }^{2}$ it appears that some special risk factors operate in spinal cord injured patients. These include forced high fluid intake, low solute intake/excretion, decreased glomerular filtration rate, resetting of the osmostat and either an absolute increase in circulating $\mathrm{ADH}$ levels or heightened renal sensitivity to $\mathrm{ADH}$. Leehey et al ${ }^{1}$ hypothesised that hypoosmolar hyponatremia in tetraplegic patients is related to decreased renal water excretion due to both intrarenal and arginine vasopressin dependent mechanisms (resetting of the osmostat) coupled with a habitually increased fluid intake. Both mechanisms of impaired water excretion may be related to a decrease in central or effective blood volume. We undertook a retrospective study of patients with hyponatremia treated at this centre over the past 9 years in order to determine the predisposing factors for hyponatremia in tetraplegics/paraplegics and to review the current trends in prevention and management of hyponatremia in this special category of patients.

\section{Methods}

This is a retrospective study of patients admitted to the Regional Spinal Injuries Centre, Southport between 1984 and 1993 who had plasma sodium levels of less than $130 \mathrm{mmol} / 1$. The details regarding their underlying illness, precipitating factors, if any, for the hyponatremic episodes, presenting clinical features, laboratory investigations, treatment provided for hyponatremia and the outcome were noted.

Nineteen patients (10 males, nine females) developed hyponatremia, of whom six developed more than one episode $(31.6 \%)$. Four of these people developed two episodes of hyponatremia and one patient developed four episodes of hypo- natremia (Tables I, II). Five of these six people with more than one episode were females. The time interval between these successive episodes of hyponatremia was less than a month in two people, and between 6 months and 5 years in the remaining four. Of the 19 patients with hyponatremia, 14 were tetraplegic and only five were paraplegic. Amongst those who were paraplegic, four had a thoracic lesion and only one had a lumbar lesion. Thus hyponatremia is infrequently seen in paraplegic patients and is very uncommon in a paraplegic patient with a lumbar lesion. The time interval between the onset of paralysis and the occurrence of the first episode of hyponatremia varied from 1 week to 37 years, but it was less than a month in only four $(21 \%)$. Thus the majority of these patients developed hyponatremia after they recovered from the acute effects of their initial illness. Six people amongst the 19 were asymptomatic during the first episode of hyponatremia. In contrast, only one of the six who developed more than one episode of hyponatremia was asymptomatic during the second or successive episode of hyponatremia.

Acute chest infection was the main precipitating factor for the development of hyponatremia in eight episodes, an acute urinary tract infection was responsible for three episodes of hyponatremia, and an infected/ gangrenous pressure sore for two episodes. Thus sepsis appears to trigger excessive secretion of $\mathrm{ADH}$ in these people. $\mathrm{Al}$ though patients were asymptomatic during seven of the $28(25 \%)$ episodes of hyponatremia, they presented with symptoms related to central nervous system dysfunction, such as confusion, incoherent speech, lethargy and drowsiness, during nine of the $28(32 \%)$ episodes (Tables I, II). The lowest plasma sodium level observed was $93 \mathrm{mmol} / \mathrm{l}$. It was less than $120 \mathrm{mmol} / \mathrm{l}$ in six episodes of hyponatremia. A female tetraplegic patient had four episodes of hyponatremia between 1984 and 1990 and she recovered fully from each episode of hyponatremia without any sequalae.

The mainstay of management was treatment of sepsis with antibiotics. Ancillary measures included fluid restriction, admin- 
Table I Clinical profile of patients with hyponatremia

\begin{tabular}{|c|c|c|c|c|c|c|c|}
\hline $\begin{array}{l}\text { Serial } \\
\text { No. }\end{array}$ & Dob & Sex & Diagnosis & $\begin{array}{l}\text { Date } \\
\text { injury/ } \\
\text { onset } \\
\text { paralysis }\end{array}$ & $\begin{array}{l}\text { Neurology } \\
\text { level }\end{array}$ & $\begin{array}{c}\text { Date } \\
\text { hypoatremia } \\
\text { observed }\end{array}$ & $\begin{array}{l}\text { Presenting clinical } \\
\text { symptoms }\end{array}$ \\
\hline 1 & 10.01 .27 & $\mathrm{M}$ & Paget's disease & 07.12 .80 & T12 & 12.10 .84 & Asymptomatic \\
\hline 2 & 10.07 .22 & $\mathrm{~F}$ & $\begin{array}{l}\text { Cystadeno carcinoma in both ovaries. } \\
\text { Squamous cell carcinoma in forchead }\end{array}$ & 01.07 .82 & $\mathrm{C} 4$ & 02.06 .88 & $\begin{array}{l}\text { Incoherent speech, confused } \\
\text { Generalised oedema }\end{array}$ \\
\hline 3 & $"$ & " & & $"$ & $"$ & 20.07 .90 & Generalised oedema \\
\hline 4 & 02.03 .24 & $\mathrm{~F}$ & Poliomyelitis & 14.12 .86 & $\mathrm{C} 5$ & 29.08 .88 & Confusion + \\
\hline 5 & 24.02 .18 & $\mathrm{~F}$ & Traumatic paraplegia & 17.11 .67 & $\mathrm{~T} 10$ & 24.02 .87 & Pyrexia, cough \\
\hline 6 & 09.01 .22 & $\mathrm{M}$ & Traumatic tetraplegia & 03.11 .80 & C6 & 26.11 .90 & Abdominal distension \\
\hline 7 & 06.08 .34 & $\mathrm{M}$ & Traumatic tetraplegia & 22.11 .91 & $\mathrm{C} 4$ & 13.12 .91 & Asymptomatic \\
\hline 8 & 13.03 .47 & $\mathrm{~F}$ & Multiple sclerosis (incomplete tetraplegia) & 1975 & $\mathrm{C} 5$ & 26.07 .93 & Asymptomatic \\
\hline 9 & " & $\mathrm{F}$ & & " & " & 03.09 .88 & Vomiting \\
\hline 10 & 21.07 .38 & $\mathrm{M}$ & Traumatic tetraplegia & 07.06 .93 & $\mathrm{C} 7$ & 17.06 .93 & Fever with chills \\
\hline 11 & $19 .(02.29$ & $\mathrm{M}$ & Traumatic paraplegia & 15.08 .56 & L1 & 29.07 .93 & Nil \\
\hline 12 & 04.07 .42 & $\mathrm{M}$ & Traumatic tetraplegia & 10.02 .90 & $\mathrm{C} 4$ & 28.07 .91 & $\begin{array}{l}\text { Features of acutc chest } \\
\text { infection } \\
\text { Collapse consolidation of } \\
\text { left lung }\end{array}$ \\
\hline $\begin{array}{l}13 \\
14\end{array}$ & $\begin{array}{c}30.03 .76 \\
n\end{array}$ & $\underset{\prime \prime}{\mathrm{M}}$ & Traumatic tetraplegia & $\underset{05.09 .92}{\prime \prime}$ & $\begin{array}{l}\text { T9 } \\
" \prime\end{array}$ & $\begin{array}{l}04.03 .93 \\
25.03 .93\end{array}$ & Lethargic \\
\hline 15 & 09.08 .20 & $\mathrm{~F}$ & Post cervical discectomy, ventilator dependent & March 1993 & $\mathrm{C} 4$ & 06.08 .93 & Aym?tomatic \\
\hline 16 & & $\mathrm{~F}$ & & & $"$ & 23.08 .93 & Generalised oedema \\
\hline 17 & 11.02 .43 & $\mathrm{M}$ & Traumatic tetraplegia & 04.09 .81 & $\mathrm{C} 4$ & 28.09 .87 & $\begin{array}{l}\text { Incomprehensive speech, } \\
\text { drowsy }\end{array}$ \\
\hline
\end{tabular}


Table I (cont)

\begin{tabular}{|c|c|c|c|c|c|c|c|}
\hline $\begin{array}{l}\text { Serial } \\
\text { No. }\end{array}$ & Dob & Sex & Diagnosis & $\begin{array}{l}\text { Date } \\
\text { injury/ } \\
\text { onset } \\
\text { paralysis }\end{array}$ & $\begin{array}{l}\text { Neurology } \\
\text { level }\end{array}$ & $\begin{array}{c}\text { Date } \\
\text { hypoatremia } \\
\text { observed }\end{array}$ & $\begin{array}{l}\text { Presenting clinical } \\
\text { symptoms }\end{array}$ \\
\hline 18 & 30.09 .27 & $\mathrm{~F}$ & Traumatic tetraplegia & 23.04 .76 & C6 & 13.08 .91 & Confused, febrile \\
\hline 19 & 16.07 .23 & $\mathrm{M}$ & Traumatic tetraplegia & 08.11 .74 & $\mathrm{C} 5$ & 19.08 .87 & Asymptomatic \\
\hline 20 & 18.07 .20 & $\mathrm{~F}$ & Post-irradiation myelopathy tetraplegia & Dec 1959 & T6 & 22.03 .84 & $\begin{array}{l}\text { Breathlessness, features of } \\
\text { acute chest infection }\end{array}$ \\
\hline 21 & $"$ & $"$ & $"$ & $"$ & $"$ & 16.05 .87 & " \\
\hline 22 & $"$ & $"$ & $"$ & $"$ & $"$ & 17.08 .90 & Acute chest infection \\
\hline 23 & $"$ & $"$ & $"$ & $"$ & $"$ & 09.11 .90 & Infected pressure sore \\
\hline 24 & 08.12 .15 & $\mathrm{~F}$ & Traumatic tetraplegia & 06.08 .93 & $\mathrm{C} 5$ & 08.08 .93 & $\begin{array}{l}\text { Agitated, pulling out } \\
\text { nasogastric tube }\end{array}$ \\
\hline 25 & 25.09 .50 & M & Traumatic tetraplegia & 10.02 .93 & $\mathrm{C} 7$ & 23.08 .93 & $\begin{array}{l}\text { Convulsions, probably not } \\
\text { related to hyponatremia. } \\
\text { Known case of epilepsy } \\
\text { on phenytoin and } \\
\text { carbamazepine }\end{array}$ \\
\hline 26 & 28.05 .50 & $\mathrm{~F}$ & Traumatic tetraplegia & 24.05 .91 & C6 & 30.05 .91 & Asymptomatic \\
\hline 27 & " & $"$ & " & " & $"$ & 2.11 .92 & Asymptomatic \\
\hline 28 & " & $"$ & $"$ & $"$ & $"$ & 01.05 .93 & $\begin{array}{l}\text { Drowsy, confused, } \\
\text { incoherent speech, } \\
\text { conjunctival oedema }\end{array}$ \\
\hline
\end{tabular}


Table II Lowest plasma sodium, corresponding potassium level, precipitating factors if any for hyponatremia, treatment and outcome of patients with hyponatremia

\begin{tabular}{|c|c|c|c|c|c|c|}
\hline $\begin{array}{l}\text { Serial } \\
\text { No. }\end{array}$ & $\begin{array}{l}\text { Lowest plasma } \\
\text { sodium level } \\
\text { observed } \\
(\mathrm{mmol} / \mathrm{l})\end{array}$ & $\begin{array}{l}\text { Corresponding } \\
\text { potassium } \\
(\mathrm{mmol} / \mathrm{l})\end{array}$ & $\begin{array}{l}\text { Serum } \\
\text { creatinine } \\
(\mu \mathrm{mol} / \mathrm{l})\end{array}$ & Precipitating factors & Management & Outcome \\
\hline 1 & 125 & 4.8 & 201 & Bronchopneumonia & $\begin{array}{l}\text { Antibiotics } \\
\text { Amoxycillin } 500 \mathrm{mg} \text { q.i.d. } \\
\text { Frusemide } 40 \mathrm{mg} \text { IV }\end{array}$ & $\begin{array}{l}\text { Recovered, discharged } \\
\quad 24.10 .84\end{array}$ \\
\hline 2 & 115 & & Urea 2.6 & Acute urinary tract infection & $\begin{array}{l}\text { Fluid restriction and } \\
\text { antibiotic (ceftazidime) }\end{array}$ & $\begin{array}{l}\text { Recovered, discharged } \\
\text { 22.6.88 }\end{array}$ \\
\hline 3 & 118 & 2.4 & 46 & $\begin{array}{l}\text { Consolidation left lung- } \\
\text { lower lobe. Diuretic } \\
\text { prescribed by GP }\end{array}$ & $\begin{array}{l}2 \mathrm{~N} \text { saline } 500 \mathrm{ml}+ \\
\text { potassium } \\
\text { Frusemide } 400 \mathrm{mg}\end{array}$ & Died 21.7.90 \\
\hline 4 & 110 & 3.5 & 52 & $\begin{array}{l}\text { Acute urinary tract infection } \\
\text { Asked to drink mugs of } \\
\text { water }\end{array}$ & $\begin{array}{l}\text { Normal saline \& potassium } \\
\text { Intravenous antibiotic } \\
\quad \text { (ceftazidime) }\end{array}$ & $\begin{array}{l}\text { Recovered, discharged } \\
\quad 4.11 .89\end{array}$ \\
\hline 5 & 119 & 3.7 & Urea 9.8 & $\begin{array}{l}\text { Bronchopneumonia, sepsis } \\
\text { from gangrenous pressure } \\
\text { sore-supra anal area }\end{array}$ & $\begin{array}{l}\text { Intravenous antibiotic. } \\
\text { Cefuroxine } 750 \mathrm{mg} \text { t.d.s. } \\
\text { No fluid restriction }\end{array}$ & $\begin{array}{l}\text { Recovered, } 4 \text { months later } \\
\text { pressure sore repaired by } \\
\text { gluteal myocutaneous flap }\end{array}$ \\
\hline 6 & 112 & 2.9 & Urea 2.7 & Nil & $\begin{array}{l}\text { Intravenous normal saline } \\
\text { with potassium }\end{array}$ & $\begin{array}{l}\text { Recovered, discharged } \\
3.12 .90\end{array}$ \\
\hline 7 & 128 & 3.5 & Urea 5.9 & Nil & Nil & $\begin{array}{l}\text { Recovered spontaneously, } \\
\text { discharged } 10.4 .92\end{array}$ \\
\hline 8 & 126 & 3.8 & $41-$ & Nil & 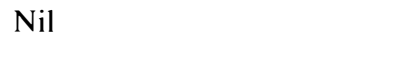 & $\begin{array}{l}\text { Recovered, discharged } \\
28.7 .93\end{array}$ \\
\hline 9 & 125 & 1.7 & Urea 3.2 & $\begin{array}{l}\text { Peritonitis due to } \\
\text { perforation of sigmoid } \\
\text { colon }\end{array}$ & $\begin{array}{l}2 \mathrm{~N} \text { saline }+\mathrm{KCl} \text {. Resection } \\
\text { of sigmoid colon on } \\
8.9 .88\end{array}$ & $\begin{array}{l}\text { Recovered, discharged } \\
\quad 19.12 .88\end{array}$ \\
\hline 10 & 119 & 3.8 & 66 & $\begin{array}{l}\text { Acute urinary tract infection } \\
\text { Low salt intake }\end{array}$ & $\begin{array}{l}\text { Antibiotics-Claforan } \\
1 \mathrm{~g} \times 8 \mathrm{~h} \\
\text { Increased salt intake in diet }\end{array}$ & $\begin{array}{l}\text { Plasma sodium gradually } \\
\text { returned to normal, } \\
\text { otherwise asymptomatic }\end{array}$ \\
\hline 11 & 123 & 3.6 & 73 & Large fluid intake. Diuretic & $\begin{array}{l}\text { Fluid restriction. Increased } \\
\text { salt intake in diet }\end{array}$ & $\begin{array}{l}\text { Recovered, discharged } \\
\text { home. }\end{array}$ \\
\hline 12 & 126 & 3.8 & Urea 2.7 & Bronchopneumonia left lung & $\begin{array}{l}\text { Bronchoscopy. } \\
\text { Antibiotics - cefotaxime } \\
\text { and aminophylline }\end{array}$ & $\begin{array}{l}\text { Recovered, discharged } \\
\text { home } 21.10 .91\end{array}$ \\
\hline
\end{tabular}


Table II (cont)

\begin{tabular}{|c|c|c|c|c|c|c|}
\hline $\begin{array}{l}\text { Serial } \\
\text { No. }\end{array}$ & $\begin{array}{l}\text { Lowest plasma } \\
\text { sodium level } \\
\text { observed } \\
(\mathrm{mmol} / \mathrm{l})\end{array}$ & $\begin{array}{l}\text { Corresponding } \\
\text { potassium } \\
(\mathrm{mmol} / \mathrm{l})\end{array}$ & $\begin{array}{l}\text { Scrum } \\
\text { creatinine } \\
(\mu \mathrm{mol} / 1)\end{array}$ & Precipitating factors & Management & Outcome \\
\hline 13 & 125 & 4.2 & 43 & $\begin{array}{l}\text { Head injury, } \\
\text { hypopituitarism. } \\
\text { ?Side effect of desmopressin }\end{array}$ & Nil specific & $\begin{array}{l}\text { Plasma sodium rose to } \\
131 \mathrm{mmol} / 118.03 .93 \mathrm{but} \\
\text { hyponatremia recurred } \\
25.3 .93\end{array}$ \\
\hline 14 & 123 & 4.1 & 62 & $\begin{array}{l}\text { Head injury, } \\
\text { hypopituitarism. } \\
\text { ?Side effect of desmopressin }\end{array}$ & $\begin{array}{l}\text { Started on hydrocortisone } \\
20 \mathrm{mg} \text { mane and } 10 \mathrm{mg} \\
\text { nocte }\end{array}$ & $\begin{array}{l}\text { Recovered, plasma sodium } \\
\text { rose to } 140 \mathrm{mmol} / \mathrm{l} \\
24.04 .93\end{array}$ \\
\hline 15 & 127 & 4.6 & 48 & Nil & Fluid restriction & Recovered \\
\hline 16 & 128 & 4.2 & 58 & Nil & $\begin{array}{l}\text { Administered } 20 \% \text { albumin } \\
\text { for hypoalbuminemia }\end{array}$ & Recovered \\
\hline 17 & 106 & 4.1 & 51 & Nil & $\begin{array}{l}\text { Hypertonic saline } \\
\text { desmopressin } 4 \text { mg IM. } \\
\text { Restricted oral fluids }\end{array}$ & $\begin{array}{l}\text { Recovered, discharged } \\
\quad 12.10 .87\end{array}$ \\
\hline 18 & 117 & 4.0 & Urea 0.7 & Acute chest infection & $\begin{array}{l}\text { Fluid restriction } \\
\text { Cefuroxime } 1.5 \mathrm{~g} / \mathrm{dl} \mathrm{IV}\end{array}$ & Recovered, discharged \\
\hline 19 & 114 & 4.1 & 66 & Nil & $\begin{array}{l}\text { Added table salt. Fluid } \\
\text { restriction DDVAP }\end{array}$ & $\begin{array}{l}\text { Recovered, discharged } \\
\text { home }\end{array}$ \\
\hline 20 & 118 & 3.7 & 54 & $\begin{array}{l}\text { Diuretic, } \\
\text { bronchopneumonia }\end{array}$ & $\begin{array}{l}\text { Diuretic continued } \\
\text { (Navidrex-K) } \\
\text { Slow sodium tablet } 1 \mathrm{t} . \mathrm{i} . \mathrm{d} \text {. } \\
\text { Fluid ( } 1500 \text { IV \& } 3400 \\
\text { oral). Antibiotic }\end{array}$ & $\begin{array}{l}\text { Recovered, discharged } \\
\text { 15.7.84 }\end{array}$ \\
\hline 21 & 93 & 2.4 & 42 & $\begin{array}{l}\text { Acute chest infection, } \\
\text { diuretic, drinking large } \\
\text { volumes of water }\end{array}$ & $\begin{array}{l}\text { Amoxycillin, chest } \\
\text { physiotheraphy with } \\
\text { terbutaline. Fluid } \\
\text { restriction, normal saline }\end{array}$ & $\begin{array}{l}\text { Recovered, discharged } \\
\text { home } 4.6 .87\end{array}$ \\
\hline 22 & 105 & 4.0 & 41 & $\begin{array}{l}\text { Bronchopneumonia, left } \\
\text { lower lobe }\end{array}$ & $\begin{array}{l}\text { Fluid restriction, normal } \\
\text { saline IV } \\
\text { Hydrocortisone } 100 \mathrm{mg} \\
\text { t.i.d. } \\
\text { Cerufoxime } 1 \mathrm{ml} 1.5 \mathrm{~g} \mathrm{IV}\end{array}$ & $\begin{array}{l}\text { Recovered, discharged } \\
\text { home } 12.11 .90\end{array}$ \\
\hline 23 & 125 & 4.1 & Urea 4.1 & $\begin{array}{l}\text { Infected ischial pressure } \\
\text { sore - cavity down to } \\
\text { bone }\end{array}$ & Blood transfusion. Dressing & Discharged home 12.11 .90 \\
\hline
\end{tabular}


Table II (cont)

\begin{tabular}{|c|c|c|c|c|c|c|}
\hline $\begin{array}{l}\text { Serial } \\
\text { No. }\end{array}$ & $\begin{array}{l}\text { Lowest plasma } \\
\text { sodium level } \\
\text { observed } \\
(\mathrm{mmol} / \mathrm{l})\end{array}$ & $\begin{array}{l}\text { Corresponding } \\
\text { potassium } \\
(\mathrm{mmol} / \mathrm{l})\end{array}$ & $\begin{array}{l}\text { Serum } \\
\text { creatinine } \\
(\mu \mathrm{mol} / \mathrm{l})\end{array}$ & Precipitating factors & Management & Outcome \\
\hline 24 & 121 & 5.0 & 96 & $\begin{array}{l}\text { Fluid overload in another } \\
\text { hospital when } \\
\text { hypotension was noticed } \\
\text { after cervical spinal injury }\end{array}$ & $\begin{array}{l}\text { Fluid restriction } \\
\text { Dopamine infusion for } \\
\text { hypotension }\end{array}$ & $\begin{array}{l}\text { Recovered from } \\
\text { hyponatremia. Plasma } \\
\text { sodium rose to } \\
132 \text { mmol/1 on } 10.8 .93 \text {. } \\
\text { She died } 13.8 .93 \text { due to } \\
\text { bronchopneumonia \& } \\
\text { respiratory insufficiency }\end{array}$ \\
\hline 25 & 124 & 4.4 & 47 & Carbamazepine & $\begin{array}{l}\text { Rectal diazepan for } \\
\text { convulsions }\end{array}$ & Recovered \\
\hline 26 & 103 & 2.9 & Urea 5.1 & Nil & Fluid restriction & Recovered \\
\hline 27 & 125 & 4.1 & 48 & Nil & Nil & Recovered \\
\hline 28 & 98 & 1.8 & 48 & $\begin{array}{l}\text { Large fluid intake. Diuretic } \\
\text { prescribed by GP }\end{array}$ & $\begin{array}{l}\text { Fluid restriction }+ \\
\text { potassium } \\
\text { Developed grand mal } \\
\text { seizure-administered IV } \\
\text { diazepam \& phenytoin IV } \\
\text { infusion. Frusemide } 10 \mathrm{mg} \\
\text { IV }\end{array}$ & $\begin{array}{l}\text { Recovered fully, discharged } \\
\text { home }\end{array}$ \\
\hline
\end{tabular}

DDA VP = Desamino-D-arginine vasopressin. 
istration of frusemide, normal saline, potassium supplement and withdrawal of the offending agent such as a diuretic. Hypertonic saline $(2 \mathrm{~N})$ was administered in two instances only.

The outcome was excellent for all but two episodes of hyponatremia. A 60 year old female with a $\mathrm{C} 4$ lesion presented with generalised oedema and a sodium level of $118 \mathrm{mmol} / \mathrm{l}$. She had consolidation of the left lower lobe of the lung and died on the second day of hospitalisation. The other death occurred in a 78 year old tetraplegic patient who developed CNS features (e.g. agitation) of hyponatremia (plasma sodium level of $121 \mathrm{mmol} / \mathrm{l})$. She recovered from hyponatremia but later succumbed to bronchopneumonia and respiratory insufficiency.

Thus the philosophy of management of sepsis (if present)-fluid restriction, frusemide and cautious use of hypertonic saline-was successful, with a mortality rate of only $3.5 \%$ for hyponatremia and $7 \%$ hospital mortality for all these patients. No patient developed central pontine myelinolysis. No patient developed respiratory insufficiency requiring ventilatory assistance.

\section{Discussion}

Asymptomatic hyponatremia generally does not require aggressive treatment. If the patient is receiving drugs which might contribute to hyponatremia, they should be discontinued if possible. Fluid restriction to less than 1 litre/day will result in a negative water balance but will achieve only a slow increase in the serum sodium concentration, rarely exceeding $1.5 \mathrm{mmol} / 24$ hours. In patients with symptomatic hyponatremia the most appropriate therapeutic regimen is hypertonic (usually $514 \mathrm{mmol} / \mathrm{l}$ ) sodium chloride $(3 \%)$, often given in conjunction with a loop acting diuretic such as frusemide. ${ }^{2}$ In patients with raised antidiuretic hormone concentrations, simultaneous administration of frusemide may be necessary to prevent circulatory overload. Patients with arterial hypoxaemia or respiratory insufficiency should be intubated and mechanically ventilated. Hypertonic $(514 \mathrm{mmol} / \mathrm{l})$ sodium chloride $(3 \%)$ should be delivered by a constant infusion pump with the absolute increase in the serum sodium concentration limited to $25 \mathrm{mmol} / \mathrm{l}$ within the initial 48 hours of treatment. The endpoint is a plasma sodium concentration which is increased by $20-25 \mathrm{mmol} / \mathrm{l}$, or has reached $130 \mathrm{mmol} / \mathrm{l}$, or resulted in an asymptomatic patient. The serum sodium concentration should not be corrected to normal values, nor should hypernatremia be allowed to develop. There has been controversy regarding the rate of correction of symptomatic hyponatremia. It was suggested that development of central pontine myelinolysis might be the result of rapid correction of chronic hyponatremia. ${ }^{3}$ Virtually all hyponatremic patients in whom cerebral palsy developed after active correction had suffered a hypoxic episode or had their serum sodium concentration corrected to either normonatremic or hypernatremic levels or increased by more than $25 \mathrm{mmol} / \mathrm{l}$ during the first 48 hours. $^{4}$ We have not exceeded $0.5 \mathrm{mmol} / \mathrm{l} /$ hour of increase in serum sodium concentration when treating symptomatic hyponatremia and we did not encounter any case of central pontine myelinolysis. Figure 1 gives the rate of correction of hyponatremia in one of our patients who had profound hyponatremia, with a serum sodium of less than 100 $\mathrm{mmol} / \mathrm{l}$. However, there are reports of over 160 patients who have undergone rapid correction (mean $1.6 \mathrm{mmol} / \mathrm{l} /$ hour) of symptomatic hyponatremia without morbidity, clearly documenting the safety and efficacy of this approach. ${ }^{2}$

Barter \& Schwartz $z^{4}$ suggested that when patients with renal failure, adrenal failure and saline depletion were excluded, there was a group of patients with hyponatremia in whom there was indirect evidence of continuing ADH secretion. They suggested the term 'syndrome of inappropirate secretion of anitdiuretic hormone' for this condition and defined it as a combination of: (1) hyponatremia with corresponding hypoosmolality of the serum and extracellular fluid; (2) continued renal excretion of sodium; (3) absence of clinical evidence of fluid volume depletion; (4) urine less than maximally dilute; (5) normal renal function; and (6) normal adrenal function. 


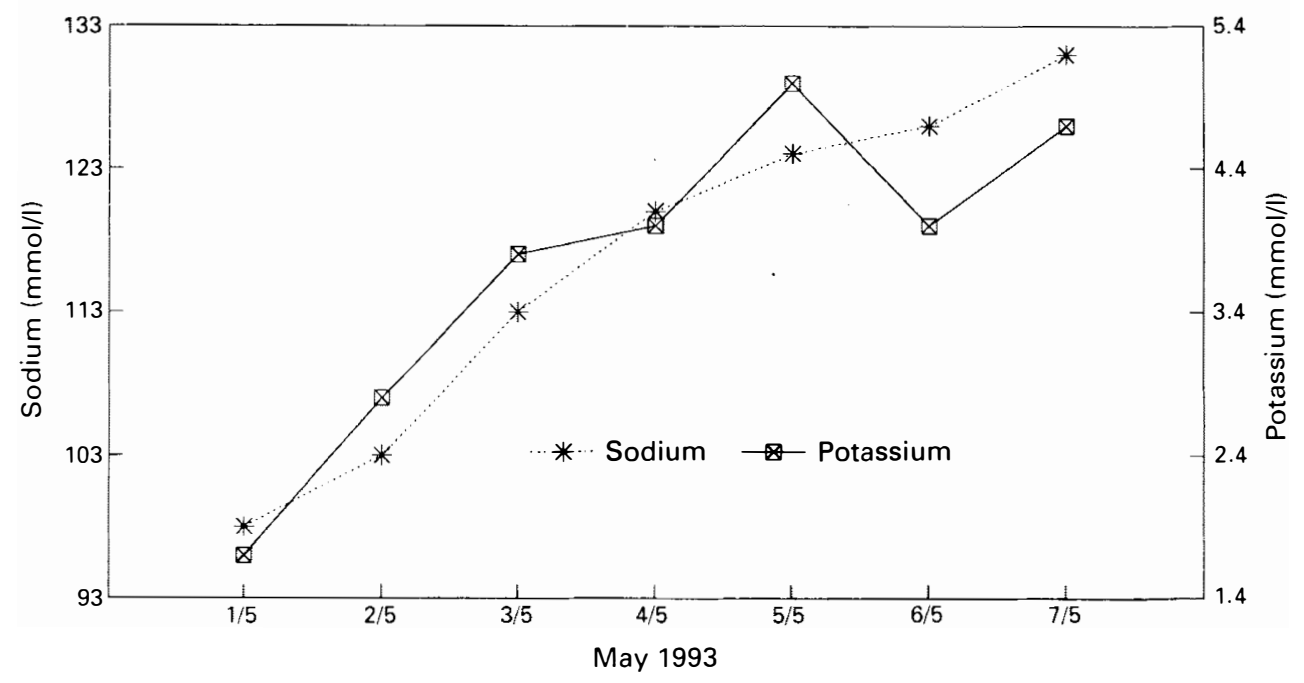

Figure 1 Rate of correction of hyponatremia in one patient.

Thomas et $a l^{6}$ described hyponatremia in four patients with chest infection with plasma arginine vasopressin (AVP) levels of $22.1,0.8,3.0$ and $46 \mathrm{pg} / \mathrm{ml}$ respectively which were excessive compared with the values expected, i.e. $<0.3 \mathrm{pg} / \mathrm{ml}$ (by extrapolation in healthy people for a plasma sodium of less than $125 \mathrm{mmol} / \mathrm{l})$. In patients with chest infection, the return of plasma sodium to normal was associated with a reduction in plasma arginine vasopressin levels. In one of the patients the AVP level was $5.6 \mathrm{pmol} / \mathrm{l}$ on 2 May 1993 when she had severe hyponatremia and a month later (9 June 1993), the AVP level was $<0.3 \mathrm{pmol} / 1$, when the plasma sodium had returned to normal. Thomas et $a l^{6}$ concluded that the hyponatremia of chest infection was transient and the increase in plasma sodium in patients with chest infection was too rapid to be produced by water-deprivation treament and was due to the return of plasma-ADH to normal. Schrier ${ }^{7}$ suggested that the release of arginine vasopressin from the pituitary due to nonosmotic stimuli is an appropriate response to stress reaction. In this study of hyponatremia in tetraplegics/ paraplegia, eight patients had an acute chest infection, three had an acute urinary infection and three suffered from sepsis either from a gangrenous pressure sore or from a perforated sigmoid colon. Hyponatremia reverted to normal, concomitant with treatment of sepsis and fluid restriction. As suggested by Thomas et al, ${ }^{6}$ instead of calling this a syndrome of inappropriate secretion of antidiuretic hormone, a simpler, more descriptive terminology such as 'hyponatremia with chest infection in a tetraplegic' would be more useful and less confusing in the clinical situation.

Sibley ${ }^{8}$ reported a case of a C5 tetraplegic patient with symptoms of acute urinary tract infection, complaints of restlessness and confusion. His serum sodium level was $118 \mathrm{mEq} / \mathrm{l}$. In this study, three patients had an acute urinary infection when they developed hyponatremia. As in hyponatremia of chest infection, these patients had probably 'inappropriate' release of $\mathrm{ADH}$, which may be an appropriate response to stress related to sepsis. With treatment of the acute urinary infection, hyponatremia also subsided in these patients.

Another mechanism which contributes to hyponatremia in cervical spinal cord injury patients is resetting of the osmostat. The osmotic threshold is defined as the plasma osmalility at which $\mathrm{ADH}$ release is initiated. When the osmotic threshold is lowered, $\mathrm{ADH}$ is released before plasma sodium or osmolality has reached normal levels. This 
mechanism has been termed 'resetting the osmostat', in which long term intake of large volumes of fluid may be implicated. Another possibility is that the lowered threshold for $\mathrm{ADH}$ release may be due to decreased sympathetic vasomotor tone.

The ability to excrete a normal fraction of an administered water load depends on the state of sodium balance. Sica \& Culpepper ${ }^{9}$ performed a water load test of $20 \mathrm{ml} / \mathrm{kg}$ bodyweight in a C6 complete male tetraplegic, who had persistent chronic hyponatremia. While on a $46 \mathrm{mmol}$ sodium diet, the minimum urinary osmolality that could be attained was only $232 \mathrm{mOsm} / \mathrm{kg}$ water and just $43 \%$ of the water load was excreted over 5 hours. The maximum free water clearance during the test was $0.43 \mathrm{ml} /$ minute. With a sodium intake of $150 \mathrm{mmol} /$ day, the urinary osmolality declined to $60 \mathrm{mOsm} / \mathrm{kg}$ water and $95 \%$ of the ingested water load was excreted in the 5 hour period. Maximum free-water clearance on a high sodium diet alone was $4.73 \mathrm{ml} /$ minute.

In our study of a tetraplegic patient, who had developed severe hyponatremia, only $14.6 \%$ of the water load was excreted in 5 hours and the lowest urine osmolality was $338 \mathrm{mOsm} / \mathrm{kg}$ water. The maximum free-water clearance was $0.44 \mathrm{ml} /$ minute. Thus, ingestion of a low salt diet results in a decreased effective extracellular fluid volume, persistent $\mathrm{ADH}$ release, and a distinctly abnormal water load test. This case illustrates the important role of sodium intake and effective extracellular volume in suppressing $\mathrm{ADH}$ release in spinal cord injury. Chronic haemodynamic instability leading to altered regulation of plasma ADH levels, when combined with a patient-specific predilection for high fluid intake and low salt intake, prediposes to the development of hyponatremia.

Leehey et $a l^{1}$ demonstrated that hypoosmolar hyponatremia in quadriplegic patients was associated with impaired water excretion. Intrarenal defects in water excretion may occur secondary to decreased osmolal clearance and/or a decrease in delivery of glomerular filtrate to the distal diluting segments of the nephron. The cause of these intrarenal defects in water excretion is not clear. Decreased osmolar clearance

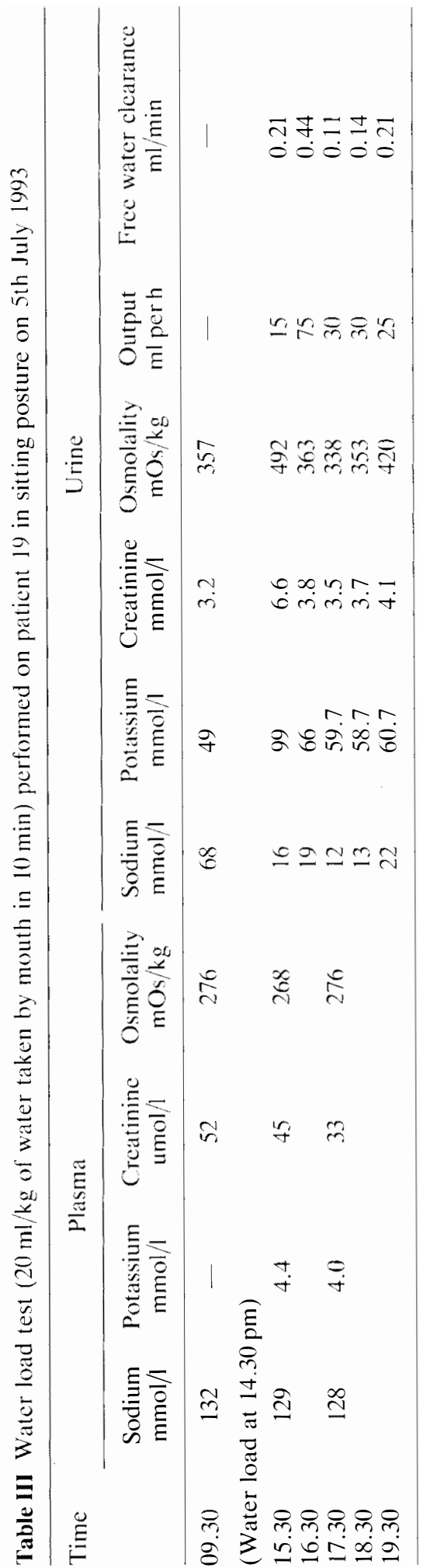


may be related to a low renal solute load, occurring either because of reduced solute intake and/or a decrease in endogenous solute production secondary to decreased muscle mass.

The reduction in the percentage of glomerular filtrate delivered to the diluting sites of the nephron could occur because of a decrease in central or effective blood volume; the resultant alterations in intrarenal haemodynamics would then cause increased fluid reabsorption in the proximal nephron segments.

We observed that the sodium intake in patient 19 was $60,75,70$ and $45 \mathrm{mmol} /$ day. Thus decreased salt intake was certainly a contributory factor to the development of hyponatremia. We also performed a water load test $(20 \mathrm{ml} / \mathrm{kg}$ body weight $)$ on her, the result of which is given in Table III. After ingestion of $1200 \mathrm{ml}$ water, the urine output for the next 5 hours was only $175 \mathrm{ml}$. She was taking normal breakfast and lunch prior to the test; thus she was not in a state of dehydration at all. Even then she excreted only $175 \mathrm{ml}$ of urine during 5 hours while she was sitting up in the chair. The phenomenon of decreased urine production in the sitting posture by tetraplegics has been observed frequently by us. The contributory factors are pooling of blood in the venous system, resulting in decreased central blood volume because of interruption of descending sympathetic pathways and absence of pumping action of leg muscles. The decrease in central or intrathoracic blood volume acts as a haemodynamic stimulus for arginine vasopressin release. Tetraplegic patients (as well as paraplegic patients with lesions above the T6 neurotome) have labile blood pressures in the low normal range and tend to develop hypotension when placed in the seated or upright tilted position. Hypotension occurring as a result of tilting in such patients not unexpectedly results in a marked elevation of plasma arginine vasopressin levels. Thus a decrease in central or effective blood volume leads to impaired water excretion due to both arginine vasopressin dependent and intrarenal mechanisms and could be the cause of both the resetting of the osmastat and the intrarenal defects in water excretion. Therefore, measures to increase effective blood volume such as salt and/or mineralocorticoid supplements and elastic leg stockings, in combination with decreased fluid intake should be beneficial in correcting the hyponatremia in spinal cord injury patients.

\section{References}

1 Leehey DJ, Picache AA, Robertson GL (1988) Hyponatremia in quadriplegic patients. Clin Sci 75: 441-444.

2 Arieff AI (1993) Management of hyponatremia. BMJ 307: 305-308.

3 Sterns RH (1987) Severe symptomatic hyponatremia: treatment and outcome. A study of 64 cases. Ann Intern Med 107: 656-664.

4 Ayus JC, Krothapalilli RK, Arieff AI (1987) Treatment of symptomatic hyponatremia and its relation to brain damage. A prospective study. $N$ Engl J Med 317: 1190-5.

5 Barter FC, Schwartz WB (1967) The syndrome of inappropriate secretion of antidiuretic hormone. Am J Med 42: 790-806.

6 Thomas TH, Morgan DB, Swaminathan R et al (1978) Severe hyponatremia. A study of 17 patients. Lancet 1: 621-624.

7 Schrier RW (1974) 'Inappropriate' v 'appropriate' anti diuretic hormone secretion. West Med J 121: 62-64.

8 Sibley P (1989) Hyponatremia in spinal cord injured persons. Rehabil Nurs 14: 29-30.

9 Sica DA, Culpepper RM (1989). Severe hyponatremia in spinal cord injury. Am J Med Sci 298: 331-333. 